\title{
Controlling cluster intermediates enables the synthesis of small PbS nanocrystals with narrow ensemble linewidths
}

Philippe B. Green, Pournima Narayanan, Ziqi Li, Philip Sohn, Christian J. Imperiale, and Mark W.B. Wilson*

Department of Chemistry, University of Toronto

Toronto, Ontario M5S3H6, Canada
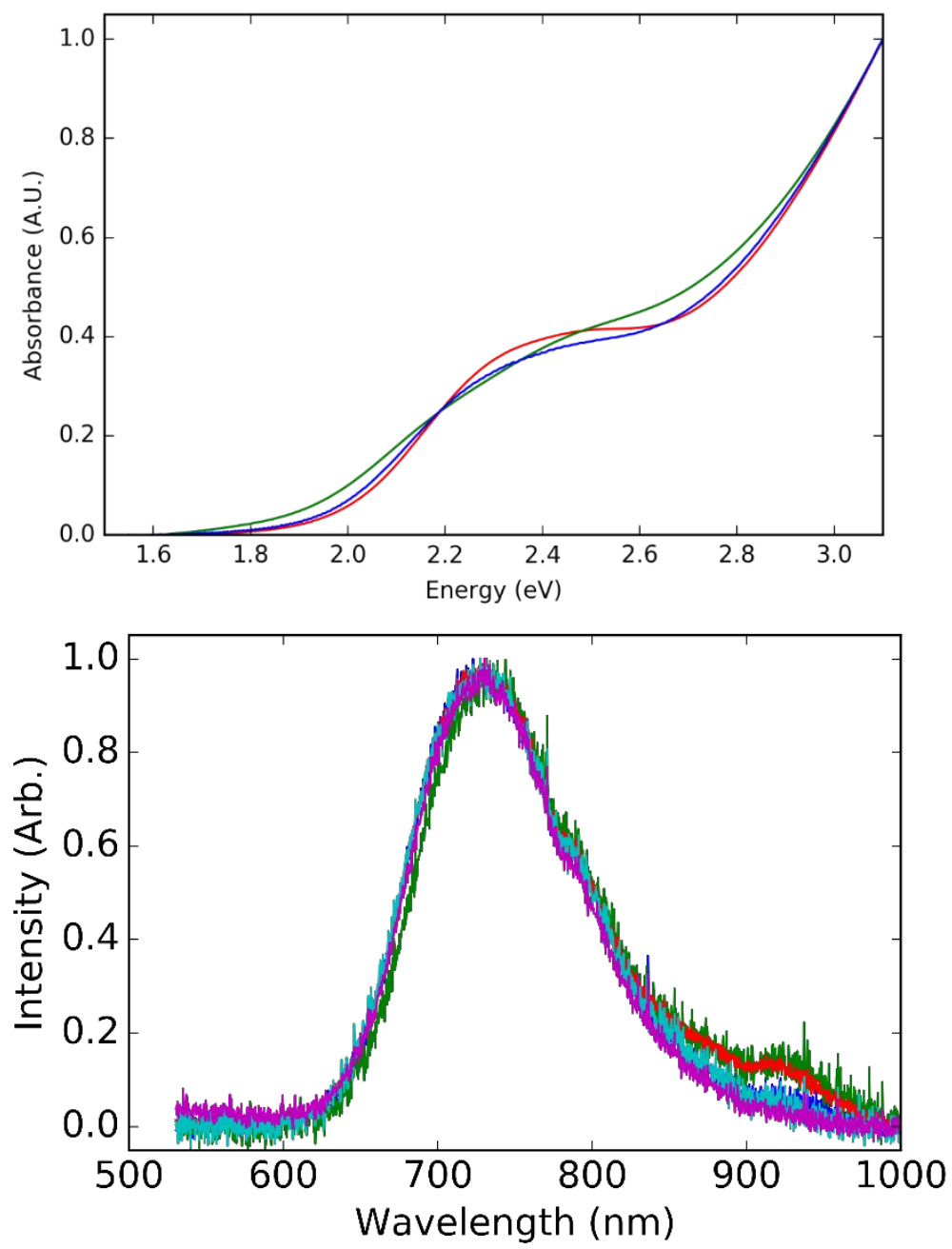

Figure S1 PNC absorbance (top, normalized at $3.1 \mathrm{eV}$ ) and emission (bottom, normalized to the peak) from a number of different synthetic batches. A similar absorbance feature is observed around $2.4 \mathrm{eV}$ for each batch, though extrinsic factors could contribute to the slight differences. These factors include the dynamics of the particular quenching process, any contamination from NCs remaining after work-up and separation (or nucleated subsequently (see Figure 2)), and any effect of the addition of the non-solvent to achieve precipitation. The emission spectrum of PNCs is even more reproducible; emission from five distinct batches are shown here. The only significant variation is slight emission near $\lambda: 920 \mathrm{~nm}$, which is consistent with emission from a small population of NCs remaining from the initial synthesis, or nucleated subsequently. (See Figure 3 ) 


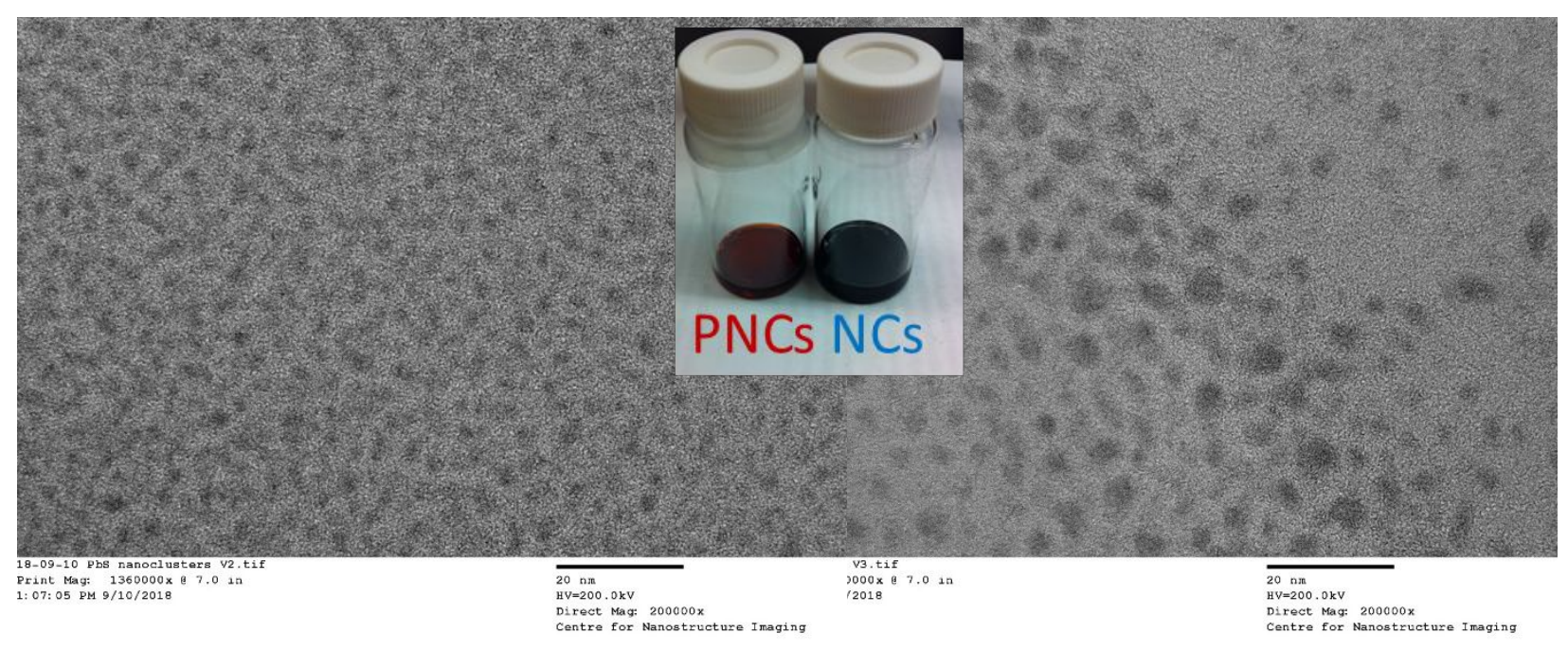

Figure S2: Transmission electron micrographs of PNCs (left) and nanocrystals (right) from the same reaction used to measure the absorption and emission spectra shown in Figure 2. The PNC image shows small particles that appear homogeneous is size. However, we were unable to obtain quantitative sizing data from this image, due to the low contrast of PNCs and the high areal PNC concentration. The paired NC image shows a wide range of sizes indicative of growth in the absence of effective size-focusing. The inset photo shows a red cluster solution and black NCs extracted from the same quenched reaction
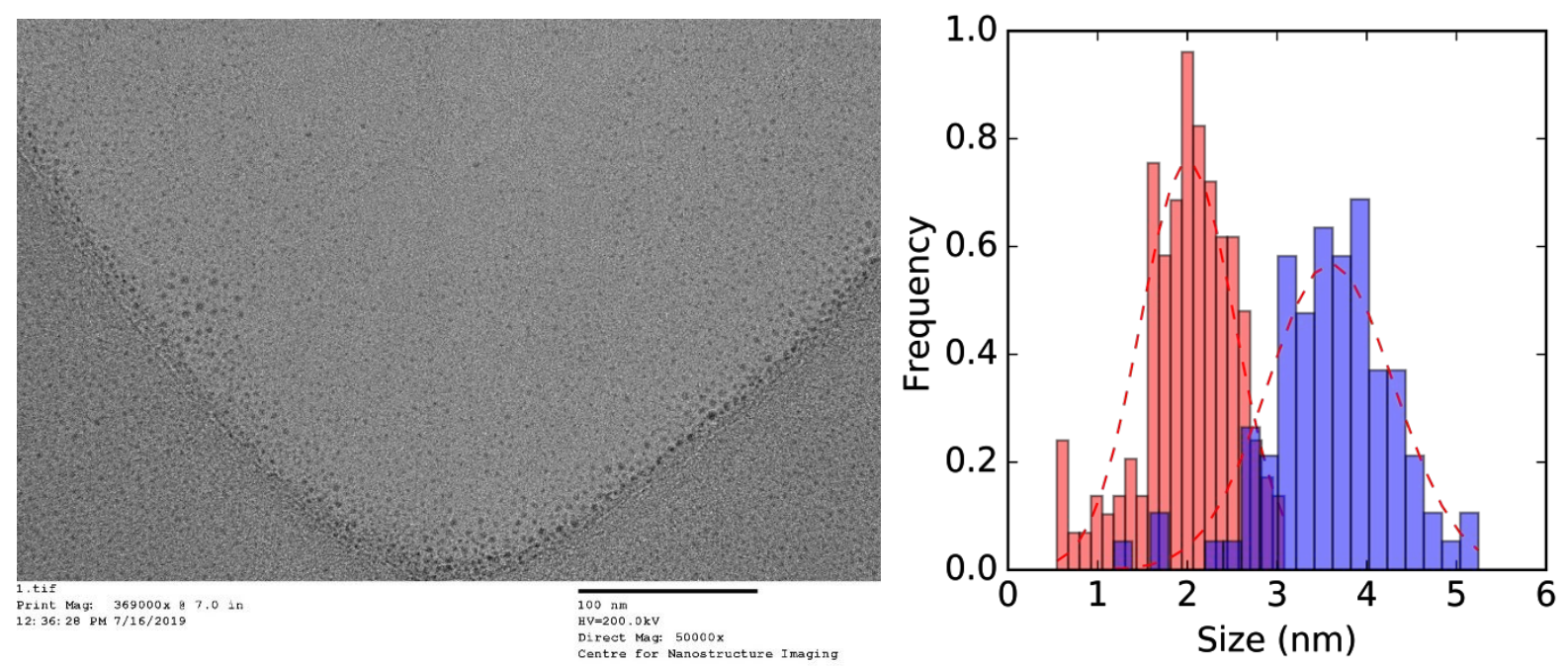

Figure S3: (Left) TEM image of the precipitate obtained from a rapidly-quenched reaction. (Right) We observe a bimodal size-distribution when comparing particles near the boundary of the grid (blue distribution) and the particles in the center (red distribution), which could be consistent either with enhanced agglomeration in local regions of high PNC concentration, or a spatial sizeselection due to flow patterns during drying. The red distribution exhibits a markedly smaller mean size $(2.0 \mathrm{~nm}$, with $26 \%$ standard deviation over 230 particles) than the blue distribution (mean size: $3.6 \mathrm{~nm}$, with $20 \%$ standard deviation over 92 particles). Given the challenge of isolating the meta-stable PNCs, the former is an estimate of their average size, though we cannot rule out that 
it is an upper limit given the possibility of cluster coalescence, and the known issues with obtaining accurate sizes using TEM for low-contrast ultra-small particles. ${ }^{1}$
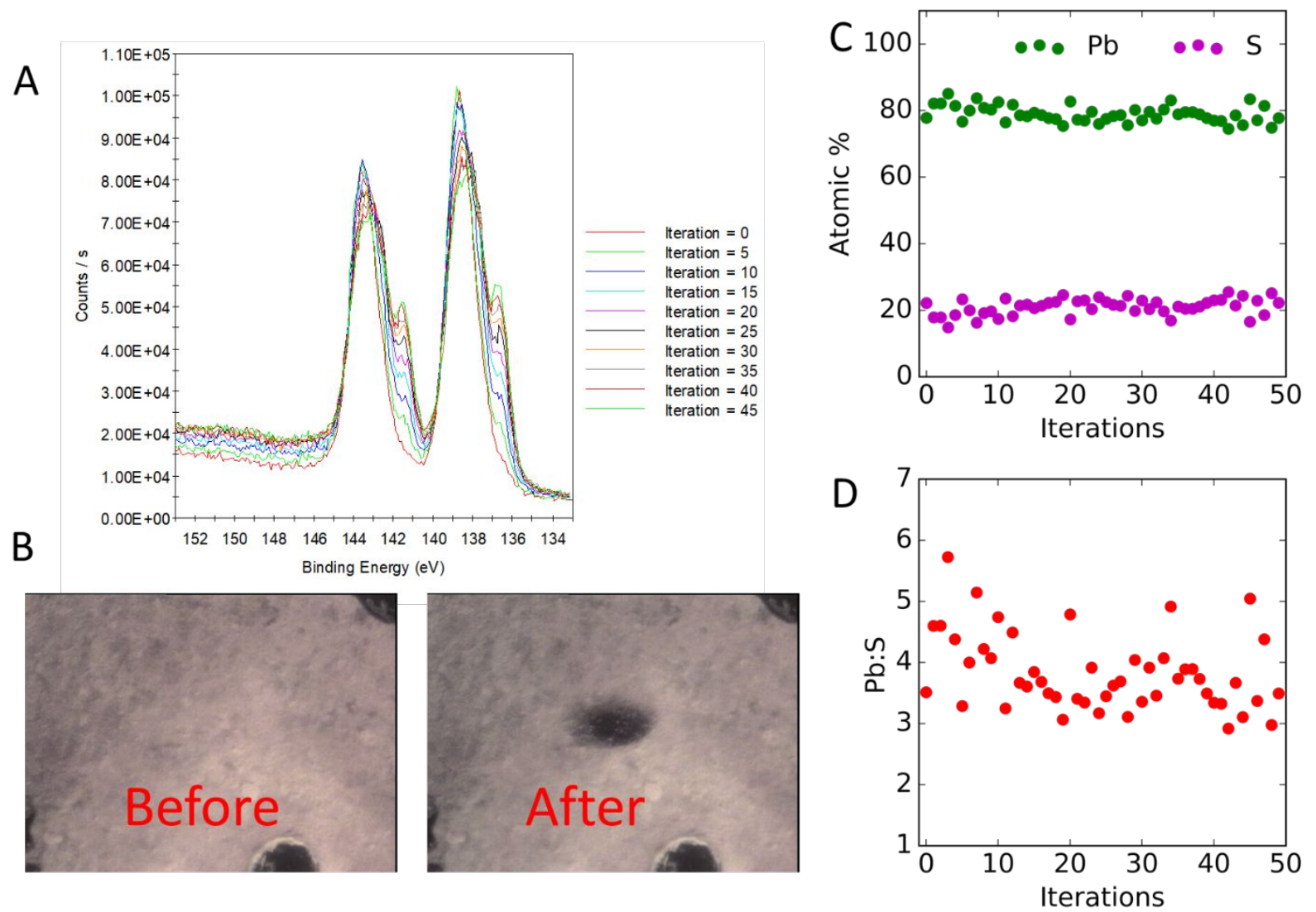

Figure S4: Data obtained using XPS on samples of PbS PNCs drop-cast on a silicon substrate. A) Representative XPS spectra taken consecutively at a single location on a single sample. The two transitions near 143 and $138 \mathrm{eV}$ correspond to emission from $\mathrm{Pb} 4 \mathrm{f} \mathrm{5/2}$ and 7/2 orbitals, respectively. 2,3 We observed changes to the XPS spectra from the PNCs during the course of measurement (each iteration took $\sim 30$ seconds), which were consistent with the production of under-charged $/$ metallic lead. ${ }^{3}$ B) These were associated with physical changes to the sample that were detectable using optical microscopy. C,D) Following convention, we estimated the $\mathrm{Pb}: \mathrm{S}$ stoichiometry of PNCs by comparing the integrated areas of the $\mathrm{Pb} 4 \mathrm{f}$ and $\mathrm{S} 2 \mathrm{~s}$ XPS peaks. ${ }^{2}$ For the representative data in $\mathrm{A}$, we found that the $\mathrm{PbS}$ : ratio was $3.8 \pm 0.1$ (mean, standard error of 50 repeated measurements to address low signal-to-noise of the sulfur peak). Despite the timedependent changes observed, the effect on the calculated $\mathrm{Pb}: \mathrm{S}$ ratio was small compared to the scatter (D). However, in measuring nine further regions across two separate samples, we only extracted values by averaging the first ten iterations, i.e. before significant changes were observable. We present all results as Table $\mathrm{S} 1$, and found that the $\mathrm{Pb}: \mathrm{S}$ ratio for PNCs was $4.0 \pm 0.2$ (mean, standard error of 10 total measurements.) 
Table S1: XPS stoichiometry from nine different spots from two different samples

\begin{tabular}{llll}
\hline Sample: Spot & $\mathbf{P b} \%$ & $\mathbf{S \%}$ & $\mathbf{P b : S}$ \\
\hline $\mathbf{1 - 1}$ & 83.14 & 16.89 & $4.9: 1$ \\
\hline $\mathbf{1 - 2}$ & 81.47 & 18.53 & $4.4: 1$ \\
\hline $\mathbf{1 - 3}$ & 81.84 & 18.16 & $4.5: 1$ \\
\hline $\mathbf{1 - 4}$ & 80.55 & 19.45 & $4.1: 1$ \\
\hline $\mathbf{2 - 1}$ & 78.55 & 21.45 & $3.7: 1$ \\
\hline $\mathbf{2 - 2}$ & 77.29 & 22.71 & $3.4: 1$ \\
\hline $\mathbf{2 - 3}$ & 80.25 & 19.75 & $4.1: 1$ \\
\hline $\mathbf{2 - 4}$ & 77.63 & 22.37 & $3.5: 1$ \\
\hline $\mathbf{2 - 5}$ & 77.39 & 22.61 & $3.4: 1$ \\
\hline
\end{tabular}

Table S2: EPMA stoichiometry from five different spots on the sample and calibration with a galena standard

\begin{tabular}{lllll}
\hline Spot & $\mathbf{P b} \%$ & $\mathbf{S \%}$ & Pb Error\% & S Error \% \\
\hline $\mathbf{1}$ & 85.57 & 14.42 & 0.82 & 3.74 \\
\hline $\mathbf{2}$ & 88.55 & 11.44 & 0.84 & 4.59 \\
\hline $\mathbf{3}$ & 85.53 & 14.46 & 0.82 & 3.74 \\
\hline $\mathbf{4}$ & 85.38 & 14.61 & 0.81 & 3.67 \\
\hline $\mathbf{5}$ & 85.09 & 14.90 & 0.83 & 3.69 \\
\hline Galena & 50.20 & 49.79 & 0.44 & 0.68
\end{tabular}

These data yielded a $\mathrm{Pb}: \mathrm{S}$ ratio for $\mathrm{PbS} \mathrm{PNC}$ of $6.2 \pm 0.4$ (mean, standard error of five measurements). 


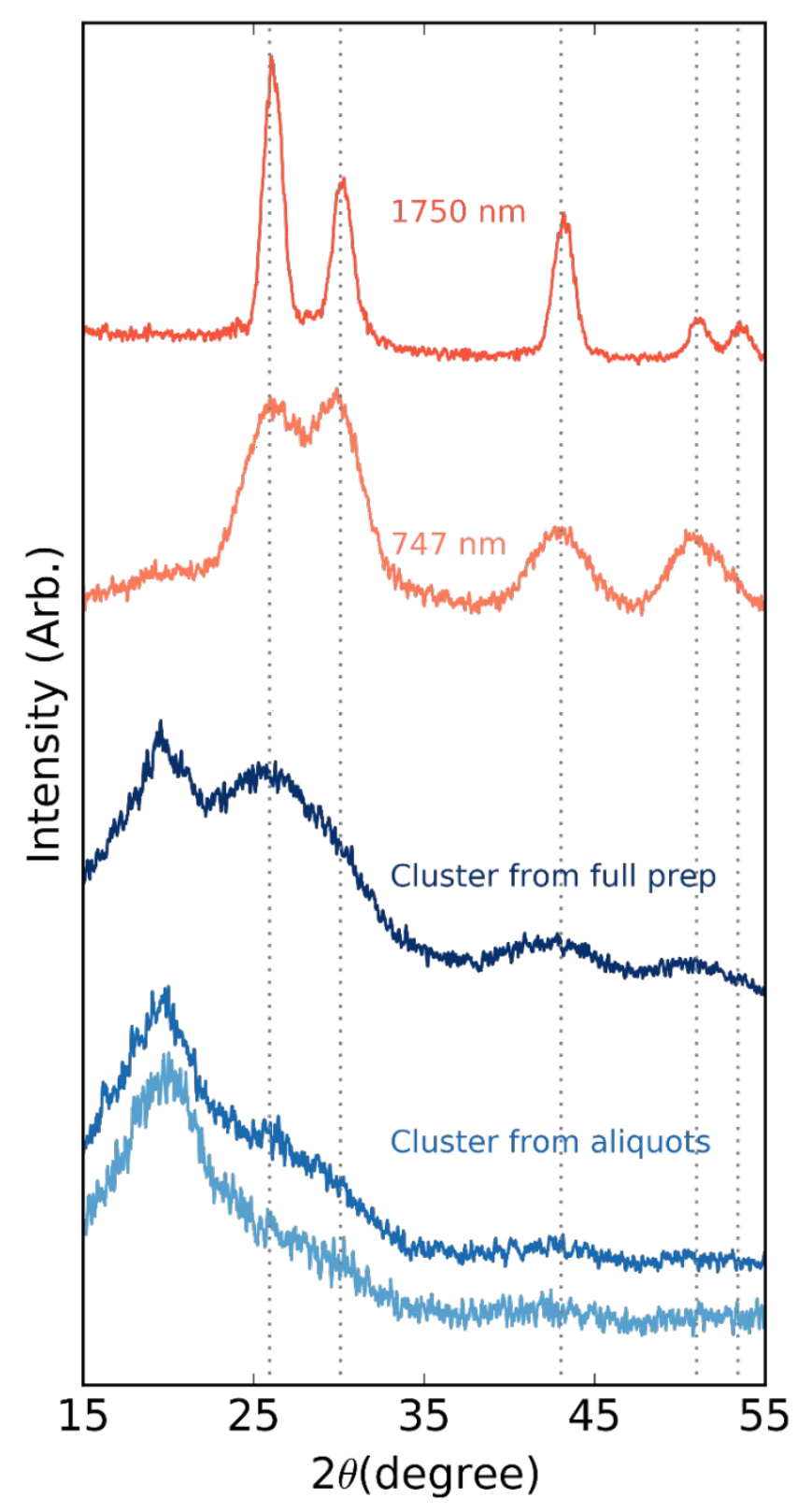

Figure S5: XRD pattern for PbS NCs and PNCs. In red are diffraction spectra of PbS NCs of two different sizes, labelled with the peak wavelength of their excitonic absorption. In blue are PNC XRDs. The two lower blue spectra were taken from aliquots extracted at the early stages of the reaction, while the upper blue spectrum was obtained by aggressively quenching the whole reaction. All three spectra exhibit a peak around $2 \theta=20^{\circ}$ that is not observed in very small $\mathrm{PbS}$ NCs. This peak has been attributed to materials with no structural order on length-scales $>2 \mathrm{~nm} .{ }^{4,5}$ However, we also observe signal from $2 \theta=25-30^{\circ}$, echoing the $\mathrm{PbS} \mathrm{NC}$ features observed above. These could reflect challenges in PNC purification, or the room-temperature evolution of the meta-stable PNCs into PbS NCs during drying. (See Figure 1 and discussion) The XRD data for the PbS NCs used as a control (red curves) was included as part of Figure S7 in the Supporting Information of a previous manuscript (Ref.7) 


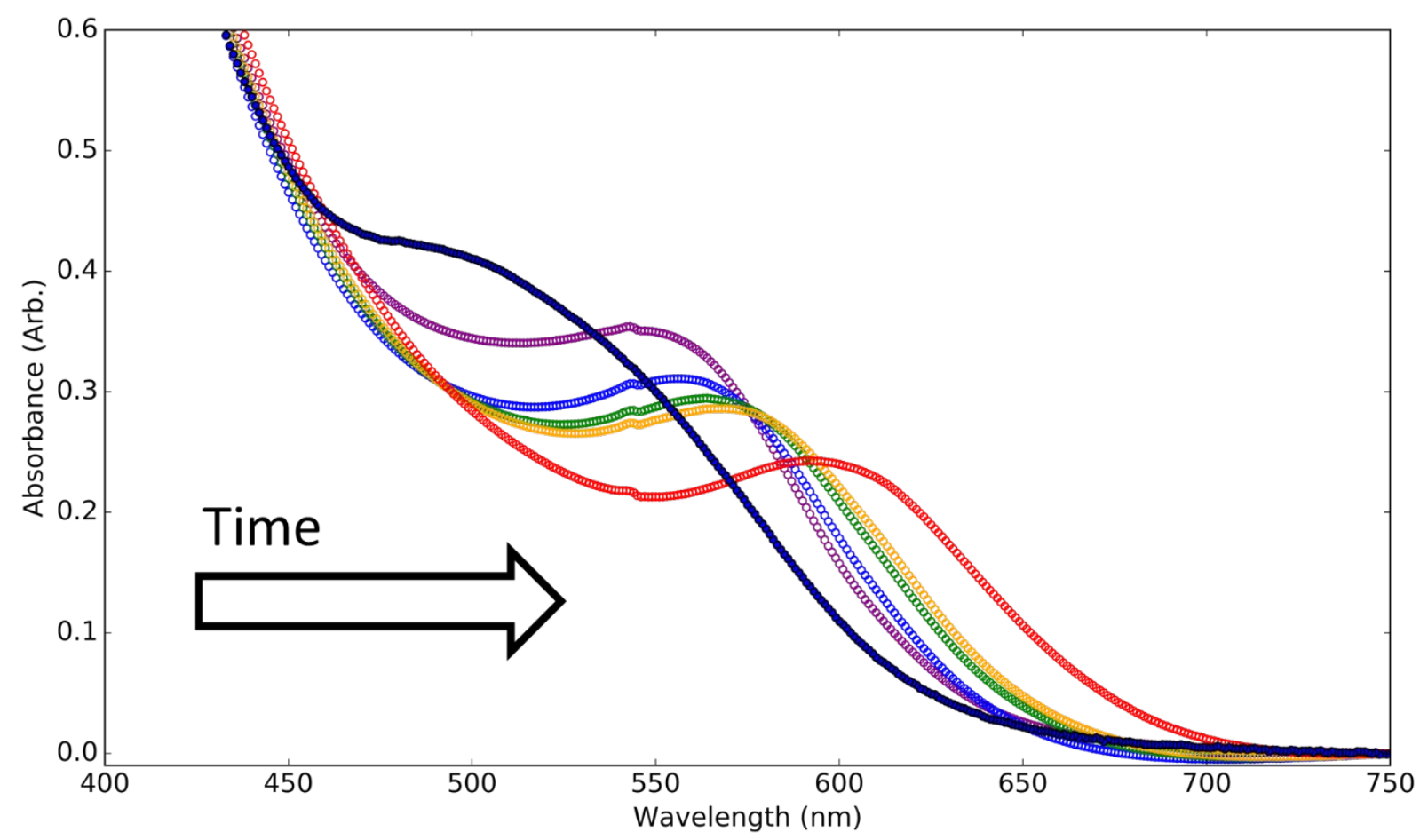

Figure S6: Time-evolution of the absorption spectra of PbS PNCs held at elevated temperatures. The dark blue curve is the absorption spectrum of the original PNC solution as extracted from a quenched reaction. (See Experimental Section for Methods) The purple curve is the absorption obtained after heating the cuvette containing the PNC solution to $85^{\circ} \mathrm{C}$, allowing it to remain for 1 minute, and then cooling the solution back to room temperature, a process that took approximately 10 minutes in total. This was repeated three additional times, talking an absorption spectrum at the end of each cycle (blue, green, orange curves, respectively). The final curve (red) was acquired after the solution was brought to $85^{\circ} \mathrm{C}$, held for five minutes, and then cooled, a process that took approximately 15 minutes overall.

Like the product observed when PNCs are held at room temperature for an extended period of time (Figure 1A,B), PbS NCs comparable to the smallest reported in literature (Figure 1) are obtained when PNCs are heated. Subsequent heating causes the excitonic peak is observed to red-shift over time, consistent with the growth of quantum-confined $\mathrm{PbS}$ NCs. After the first step-change from PNC to NC absorption (Figure 1B), we observe smooth growth, though the greater size-dispersity of these NCs is indicated by the broad absorption linewidths compared to similar-size NCs synthesized via hot injection with amines. (Figure S12) 

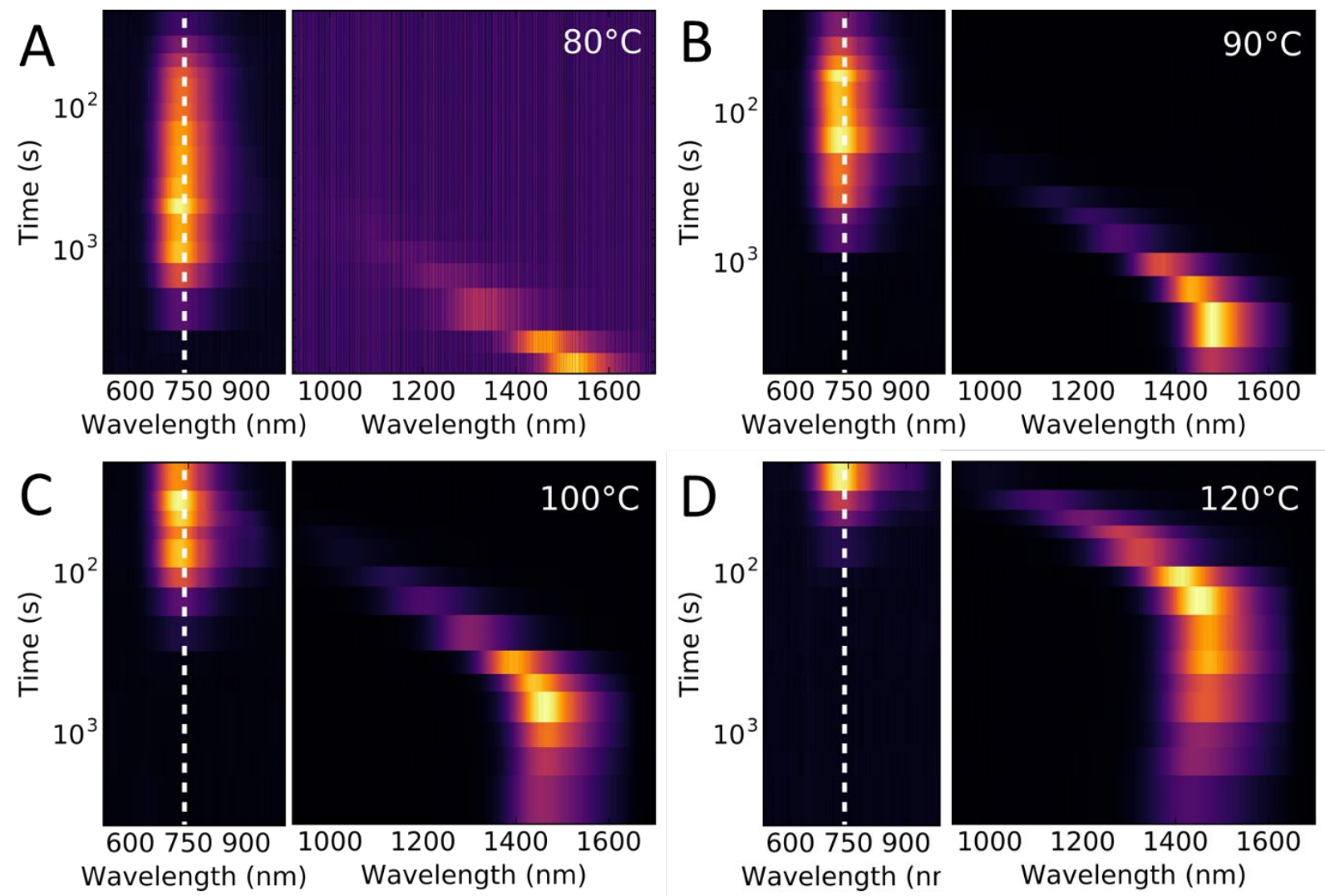

Figure S7: Temperature-dependent growth in dilute conventional reaction conditions $\left(\mathrm{A}: 80^{\circ} \mathrm{C}\right.$, $\mathrm{B}: 90^{\circ} \mathrm{C}, \mathrm{C}: 100^{\circ} \mathrm{C}, \mathrm{D}: 120^{\circ} \mathrm{C}$ ). This data was used to produce Figures $3 \mathrm{~A}-\mathrm{C}$ ) and demonstrate the changing $\mathrm{PNC}$ reaction lifetime and its effect in nanocrystal growth. 

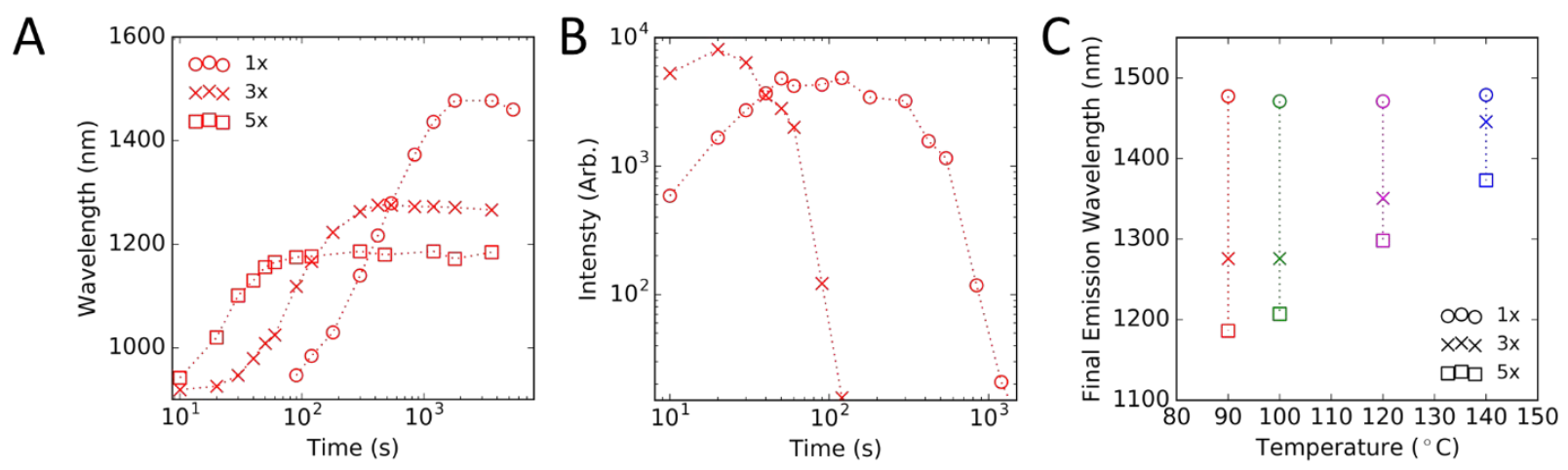

Figure S8: Concentration dependence of nanocrystal growth at different temperatures. A) Position of the peak wavelength of $\mathrm{NC}$ emission over time at $90^{\circ} \mathrm{C}$ with different concentrations of precursors. (1x (circles): reference reaction with dilute precursors, as described in the Experimental Section. 3x (diagonal crosses), 5x (squares) are reactions performed with the concentrations of $\mathrm{Pb}, \mathrm{S}$ and $\mathrm{OA}$ scaled by the given factor, so the $\mathrm{OA}: \mathrm{Pb}$ and $\mathrm{S}: \mathrm{Pb}$ ratios are unchanged.) As the concentration is increased the ultimate size of NCs at completion diminishes. Concentration also accelerates the PNC lifetime B), until the emission is undetectable given our experimentally accessible range of time-scales. C) The overall effect of temperature on the final size is dependent on the concentrations employed. In our dilute reference reaction, increasing the temperature from $90^{\circ} \mathrm{C}$ to $140^{\circ} \mathrm{C}$ has no effect on the final size. However, as we increase the concentration, we observe a growing dependence on temperature, as previously reported. ${ }^{6,7}$ 

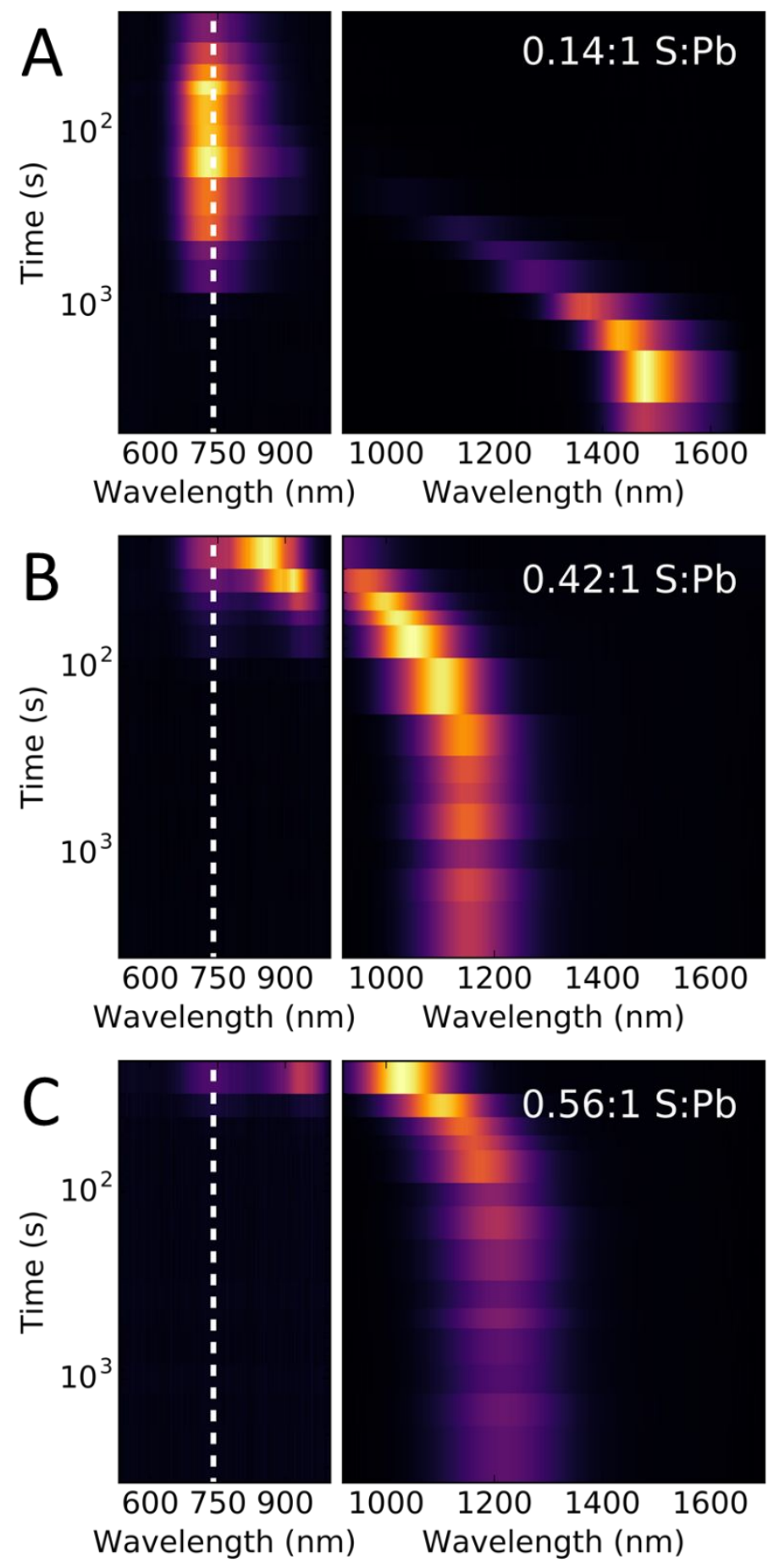

Figure S9: Dependence of the reaction kinetics on the $\mathrm{S}: \mathrm{Pb}$ ratio as monitored by the emission of aliquots taken periodically during synthesis of PbS NCs. All other synthetic conditions are as per our dilute reference reaction. (See Experimental Section for Methods) 

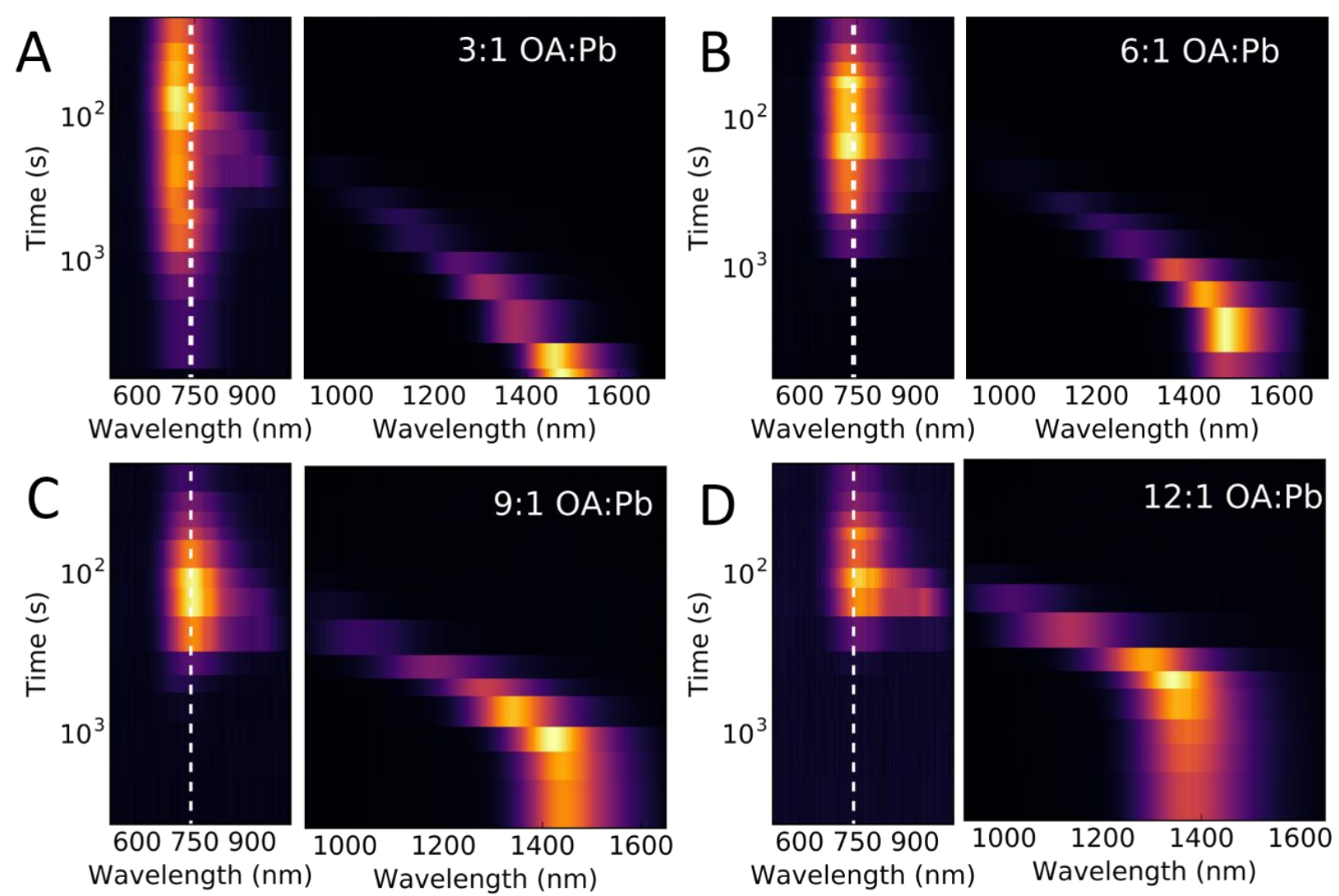

Figure S10: Dependence of the reaction kinetics on the $\mathrm{OA}: \mathrm{Pb}$ ratio as monitored by the emission of aliquots taken periodically during synthesis of PbS NCs. All other synthetic conditions are as per our dilute reference reaction. (See Experimental Section for Methods) 

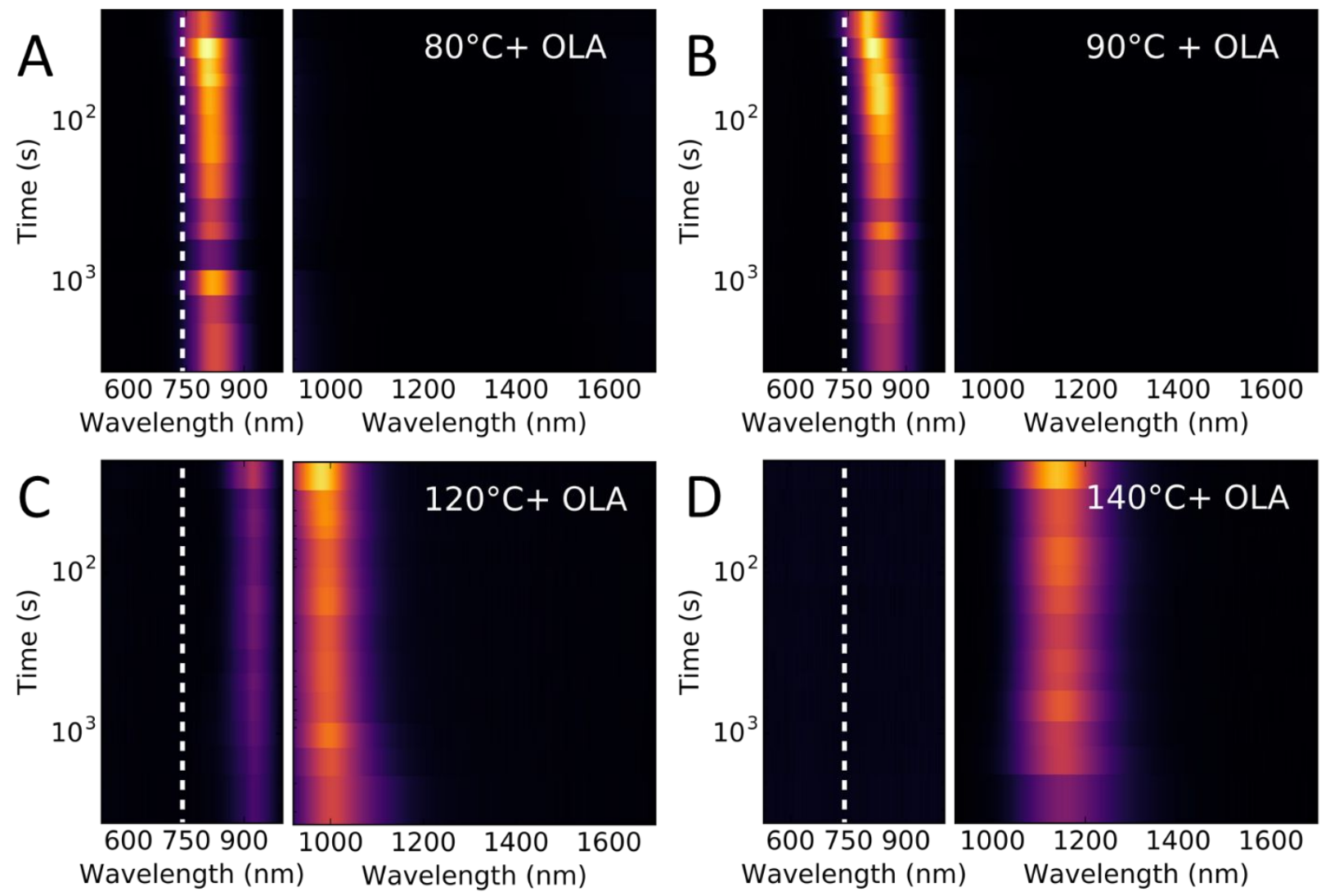

Figure S11: Dependence of the reaction kinetics on the temperature in the presence of 1.2 OLA:Pb as monitored by the emission of aliquots taken periodically during synthesis of PbS NCs. All other synthetic conditions are as per our dilute reference reaction. (See Experimental Section for Methods) 

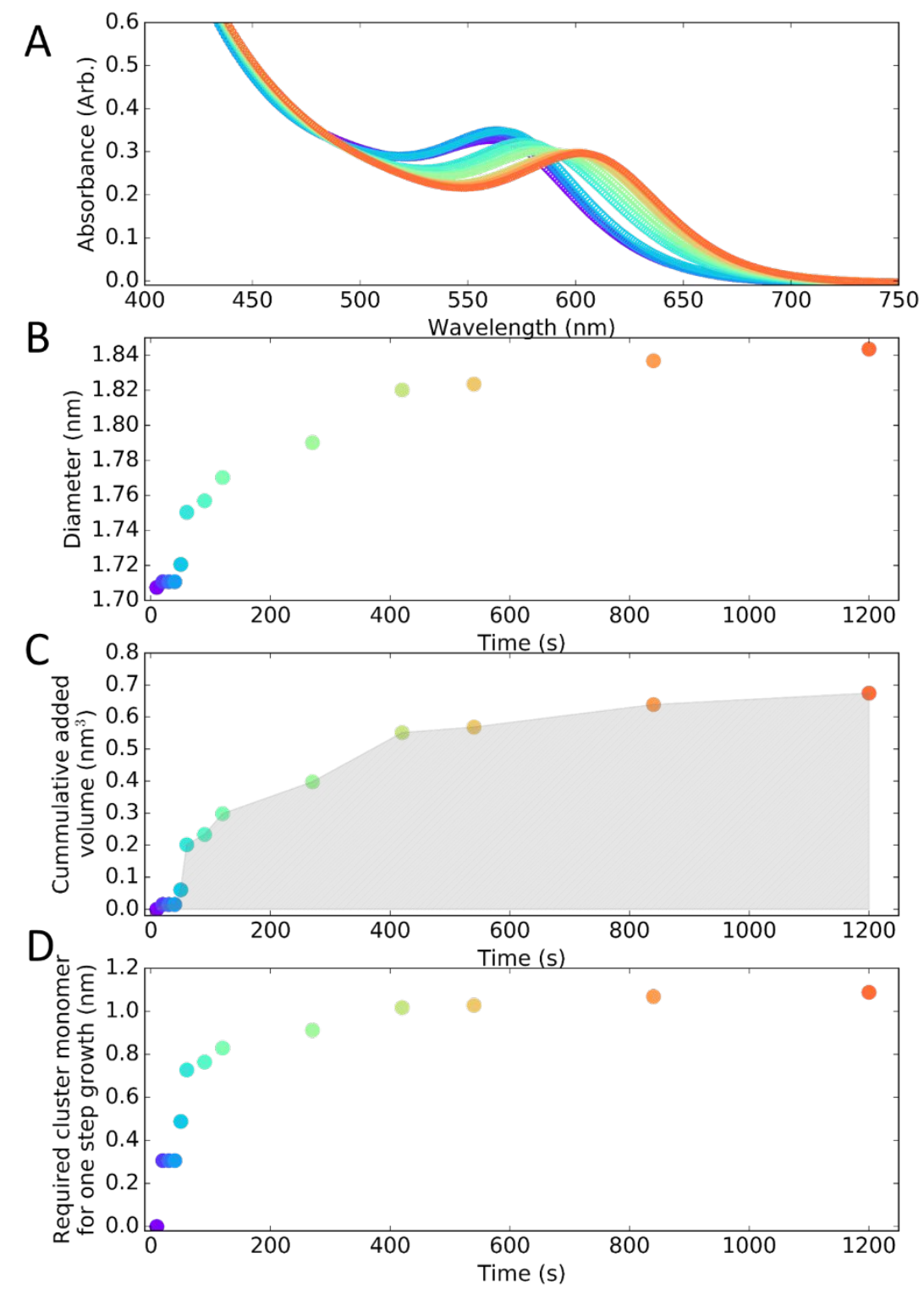

Figure S12: A: Absorption spectra (of aliquots) from growth of $\mathrm{PbS} \mathrm{NC}$ in a dilute reference reaction with amines, but at low temperature $\left(30^{\circ} \mathrm{C}\right)$ (See Experimental Section for Methods) B: Evolution of the $\mathrm{NC}$ diameter over time, calculated using the position of the first excitonic peak and a literature sizing curve ${ }^{8} \mathrm{C}$ : Cumulative added volume as compared to the $\mathrm{NC}$ observed in the first time point (at 10s post-injection) $\mathrm{D}$ : Estimated diameter of the $\mathrm{PbS}$ cluster needed to produce the observed volume increase in a single-step-addition. This shows that a single $\varnothing \sim 1 \mathrm{~nm}$ particle added directly to the initial NC would reproduce the total volume change observed. However, we see a continuous change in peak position/diameter/volume, signifying that growth proceeds via that addition of monomers contributing much less material than $\varnothing \sim 1$ nm cluster. Given our estimate of the PNC size, this data would be inconsistent with the direct addition of PNCs, but is consistent with nanocrystal growth from a classic, molecular complex-type monomer. 

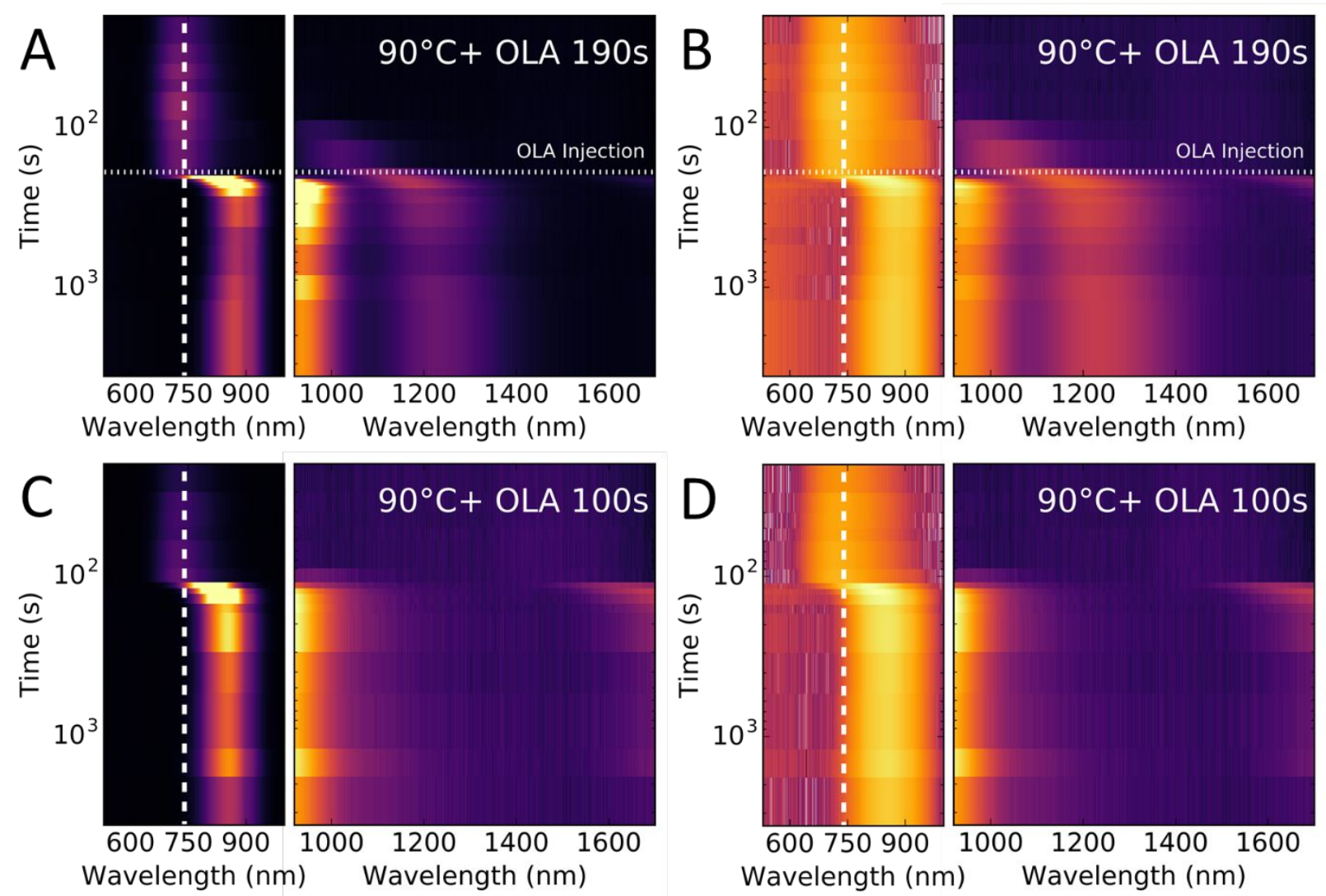

Figure S13: Mid-reaction addition of 1.2:1 OLA:Pb to conventional reactions where slow NC nucleation in the presence of PNCs had already commenced. A\&B OLA addition at 190sec (A: linear intensity, B: log intensity. The intensity of emission in each wavelength range (visible and infrared) is independently normalized to the peak value in the range.) C\&D) OLA injection at 100s (C: linear intensity, D: $\log$ intensity, scaling as above). OLA addition at times where both the PNC and $\mathrm{NC}$ are observable results in the formation of a bimodal size distribution, highlighted by the two distinct features observable after the amine injection (A,B). If no $\mathrm{NC}$ are yet observable, only one size distribution is observed (C,D). 

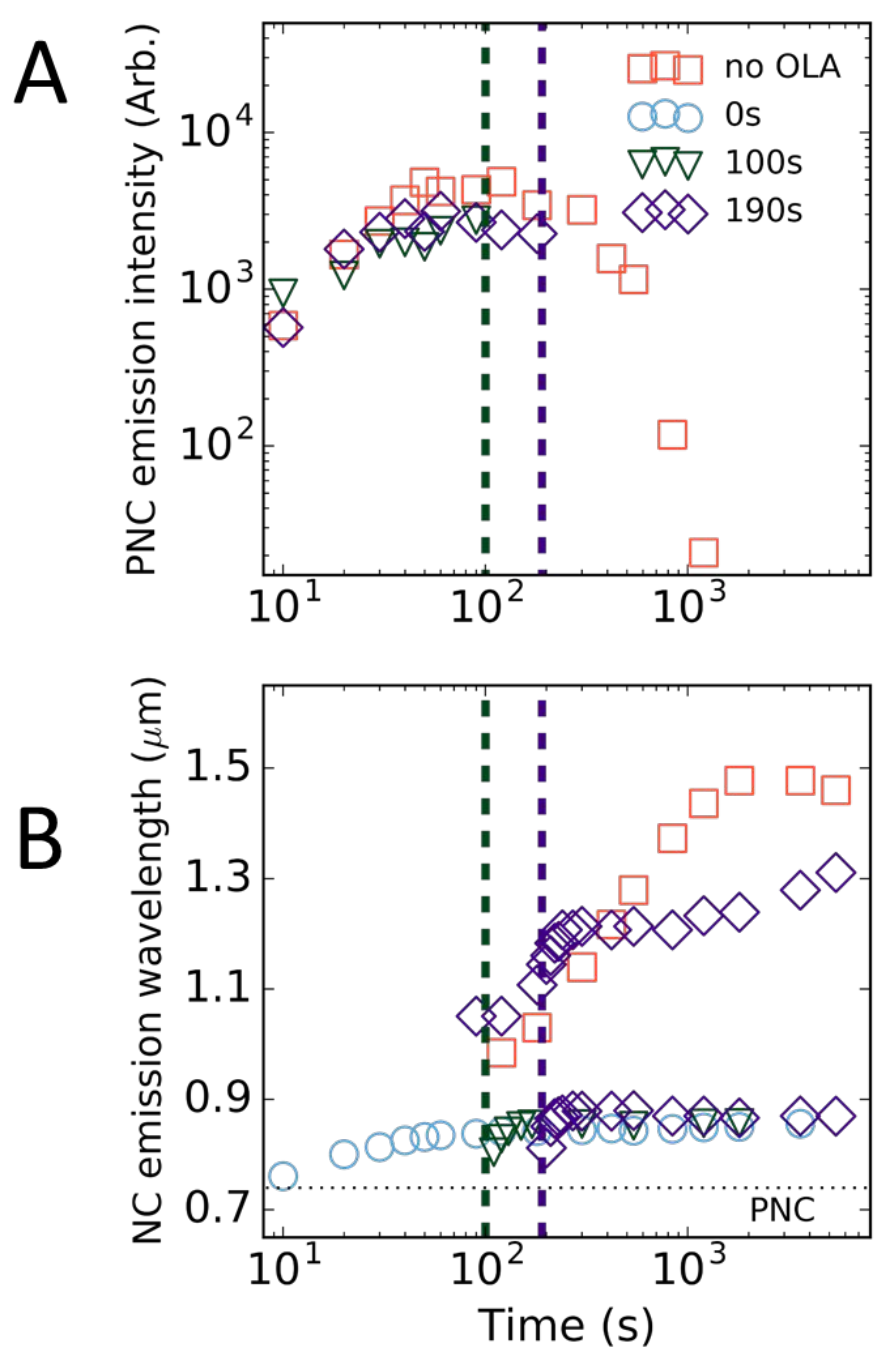

Figure S14: Effect of OLA addition during the reaction. PNC reaction profile (A) and consequent $\mathrm{NC}$ growth (B) for no OLA (red squares), OLA at $\mathrm{t}=0 \mathrm{~s}$ (blue circles), OLA at $\mathrm{t}=100 \mathrm{~s}$ (green triangles), OLA at $\mathrm{t}=190 \mathrm{~s}$ (purple diamonds). Dashed lines at $\mathrm{t}=100 \mathrm{~s}$ (green) and $\mathrm{t}=190 \mathrm{~s}$ (purple) indicate the OLA injection time. A) The PNC reaction profile, except for the reaction when OLA is present at $\mathrm{t}=0 \mathrm{~s}$, are all very similar until OLA is injected. The OLA injection results in an immediate loss of all PNC emission. B) When OLA is present at $\mathrm{t}=0 \mathrm{~s}$, NCs are immediately observable and rapidly grow to an emission feature around $860 \mathrm{~nm}$. When no OLA is added the $\mathrm{NC}$ emission is delayed and at larger emission wavelengths while a slow growth is observed. When OLA is injected at a later stage during the reaction. The reaction kinetics are taken from Figure S7B (no OLA, red squares), S11B (0s, Blue circles), S14C (100s, green triangles) and S14A (190s, purple diamond) 

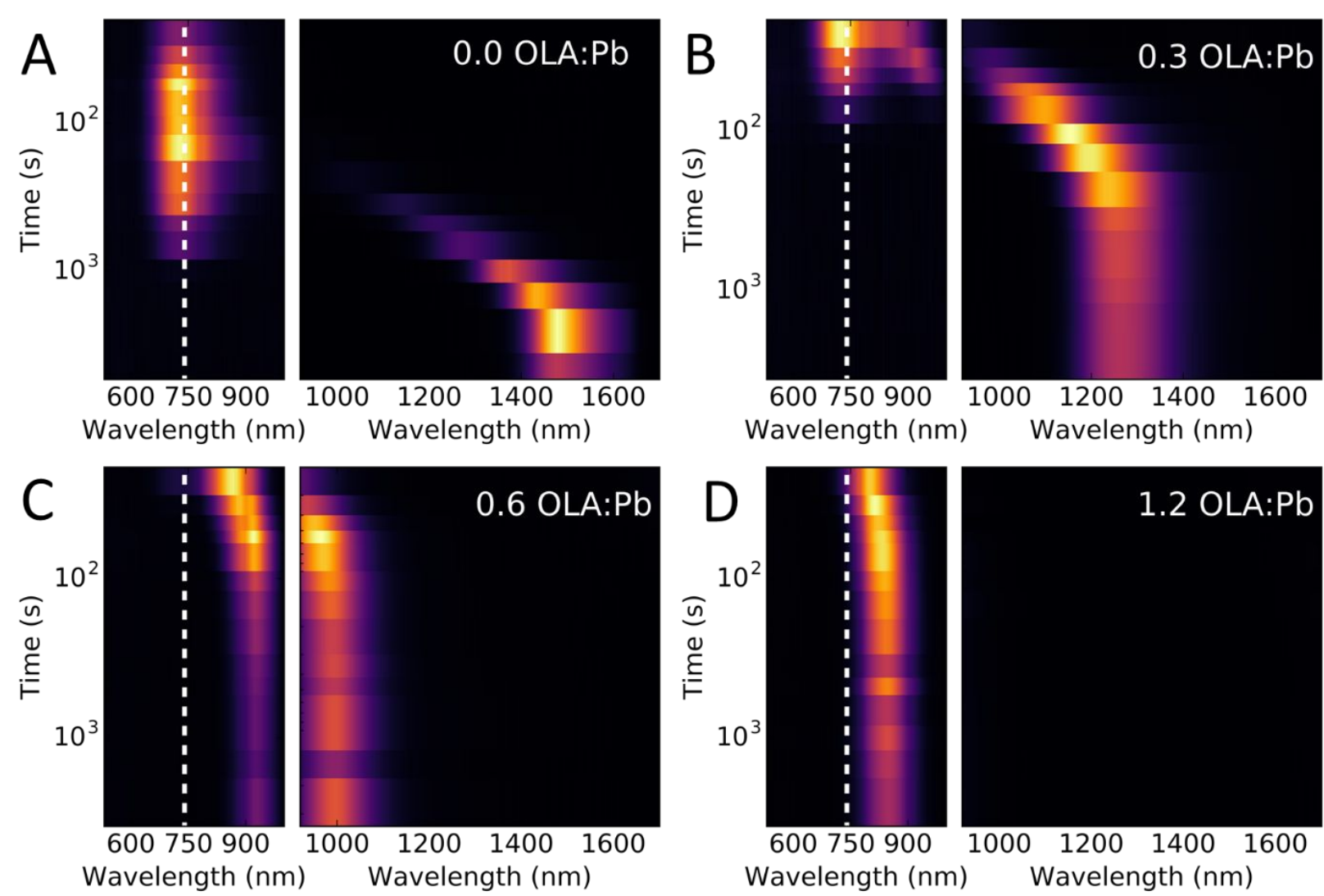

Figure S15: Dependence of the reaction kinetics on the OLA: $\mathrm{Pb}$ ratio as monitored by the emission of aliquots taken periodically during synthesis of PbS NCs. All other synthetic conditions are as per our dilute reference reaction. (See Experimental Section for Methods) 


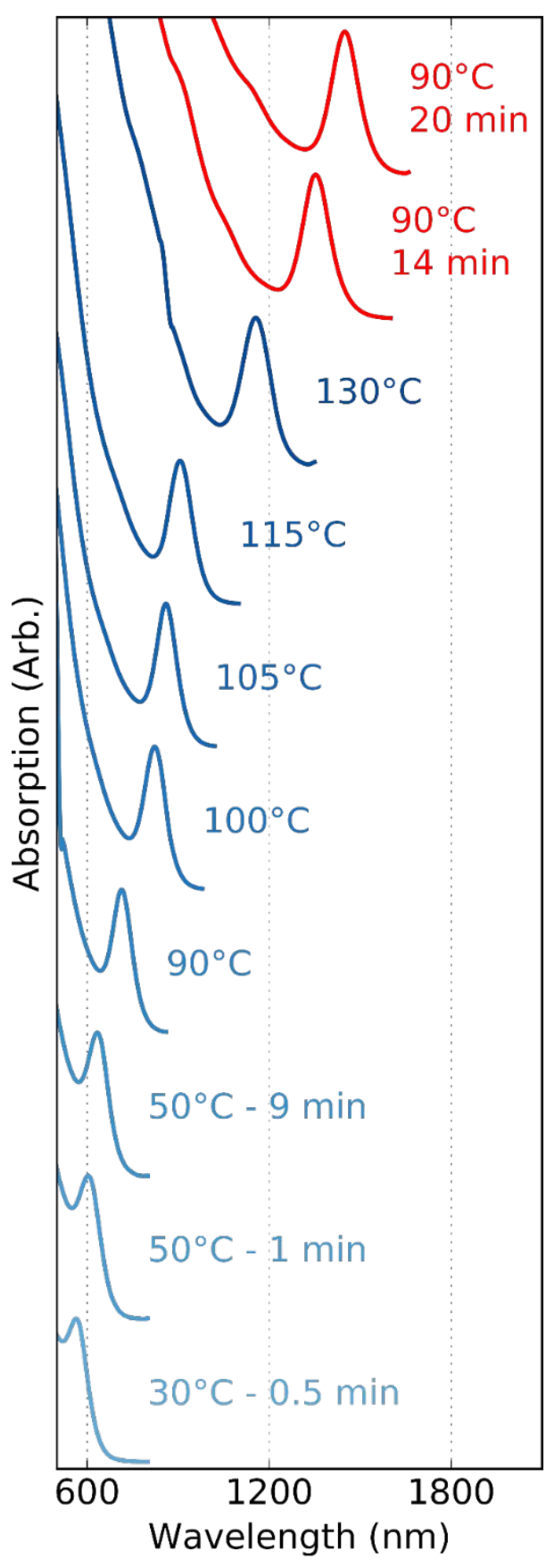

Figure S16: Absorption spectra of NCs prepared with OLA (Blue) and without (Red). OLA permits the synthesis of $\mathrm{PbS} \mathrm{NCs}$ with excitonic features with peak wavelengths as short as $\lambda_{\text {peak }, a b s}=560 \mathrm{~nm} \quad\left(\mathrm{~h} v_{\text {peak,abs }}=2.2 \mathrm{eV}\right)$, while maintaining ensemble emission linewidths that are narrow compared to previous reports. (Figure 5) The annotation indicates the temperature of the reaction and the time until the reaction was complete, and was quenched in an ice bath to forestall ripening. If no time is indicated, the rapid reaction was quenched immediately after injection. 

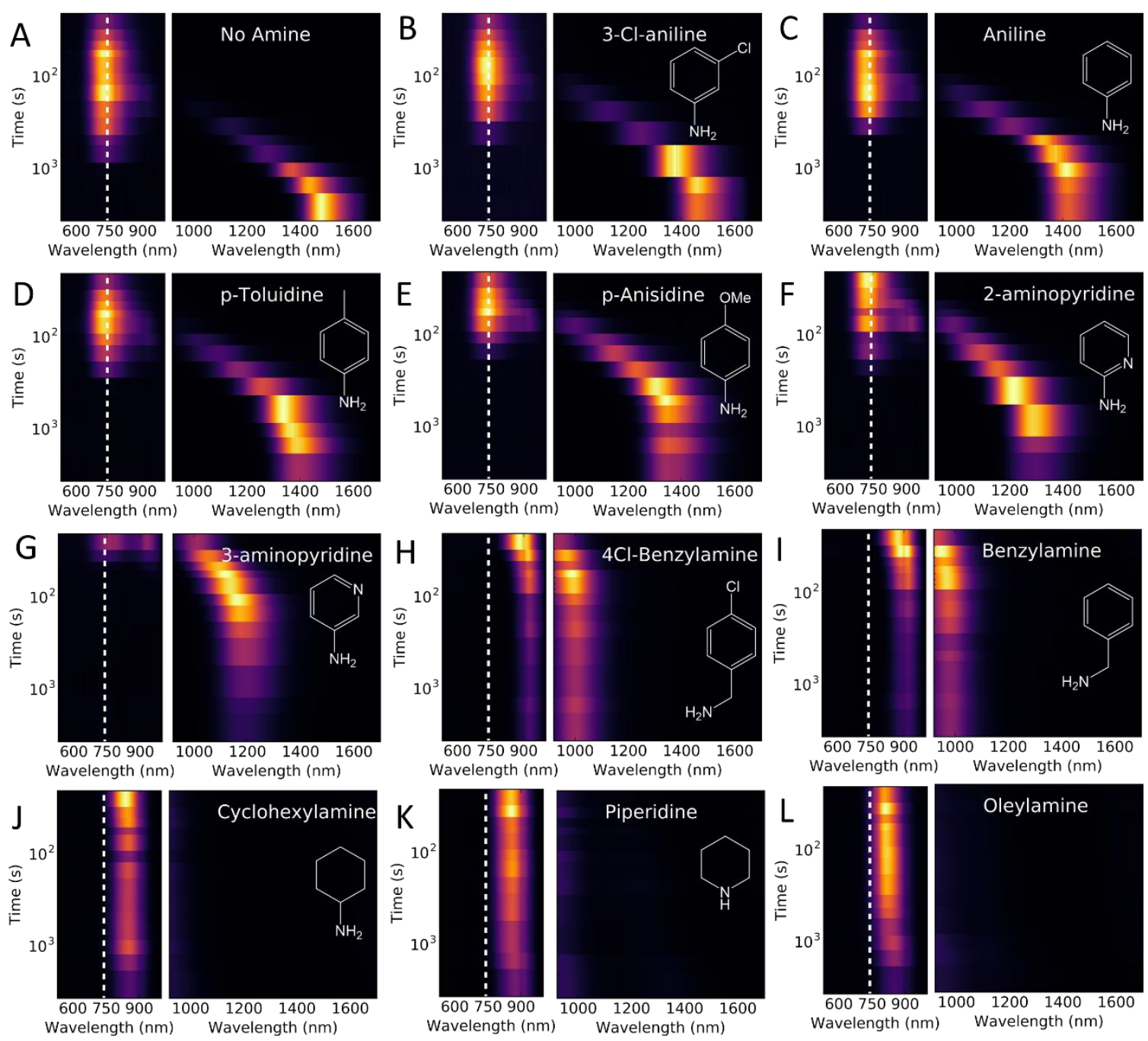

Figure S17: Influence of the primary amine $\mathrm{pKa}$ on the growth in dilute reaction coordinates (A: no amine, B: 3-Chloro-aniline, C: aniline, D: p-toluidine, E: p-anisidine, F: 2-aminopryridine, G: 3-aminopyridine, H: 4-chlorobenzylamine, I: benzylamine, J: cyclohexylamine, K: piperidine, L: oleylamine). The inset molecular structure of the amine is inserted on each figure. These data are summarized on Figure 4 in the main text. We note that 3-aminopyridine is poorly soluble in the reaction mixture, which was partially addressed by pre-heating the injection solution. 
Table S3: pKa and final emission peak position for reactions with different amines at $90^{\circ} \mathrm{C}$ 6:1 OA: Pb and 1.2 NH:Pb

\begin{tabular}{lll}
\hline Amine & pKa & $\begin{array}{l}\text { Final emission wavelength } \\
(\mathbf{n m})\end{array}$ \\
\hline No Amine & & 1480 \\
\hline 3-Chloroaniline & 3.52 & 1460 \\
\hline Aniline & 4.87 & 1419 \\
\hline p-Toluidine & 5.08 & 1395 \\
\hline p-Anisidine & 5.36 & 1350 \\
\hline 2-Aminopyridine & 6.82 & 1296 \\
\hline 3-Aminopyridine* & 6.04 & 1192 \\
\hline 4-Chlorobenzylamine & 9.14 & 1002 \\
\hline Benzylamine & 9.34 & 982 \\
\hline Cyclohexylamine & 10.64 & 865 \\
\hline Piperidine & 11.12 & 862 \\
\hline Oleylamine & 10.6 & 864 \\
\hline
\end{tabular}

*Solubility issues 

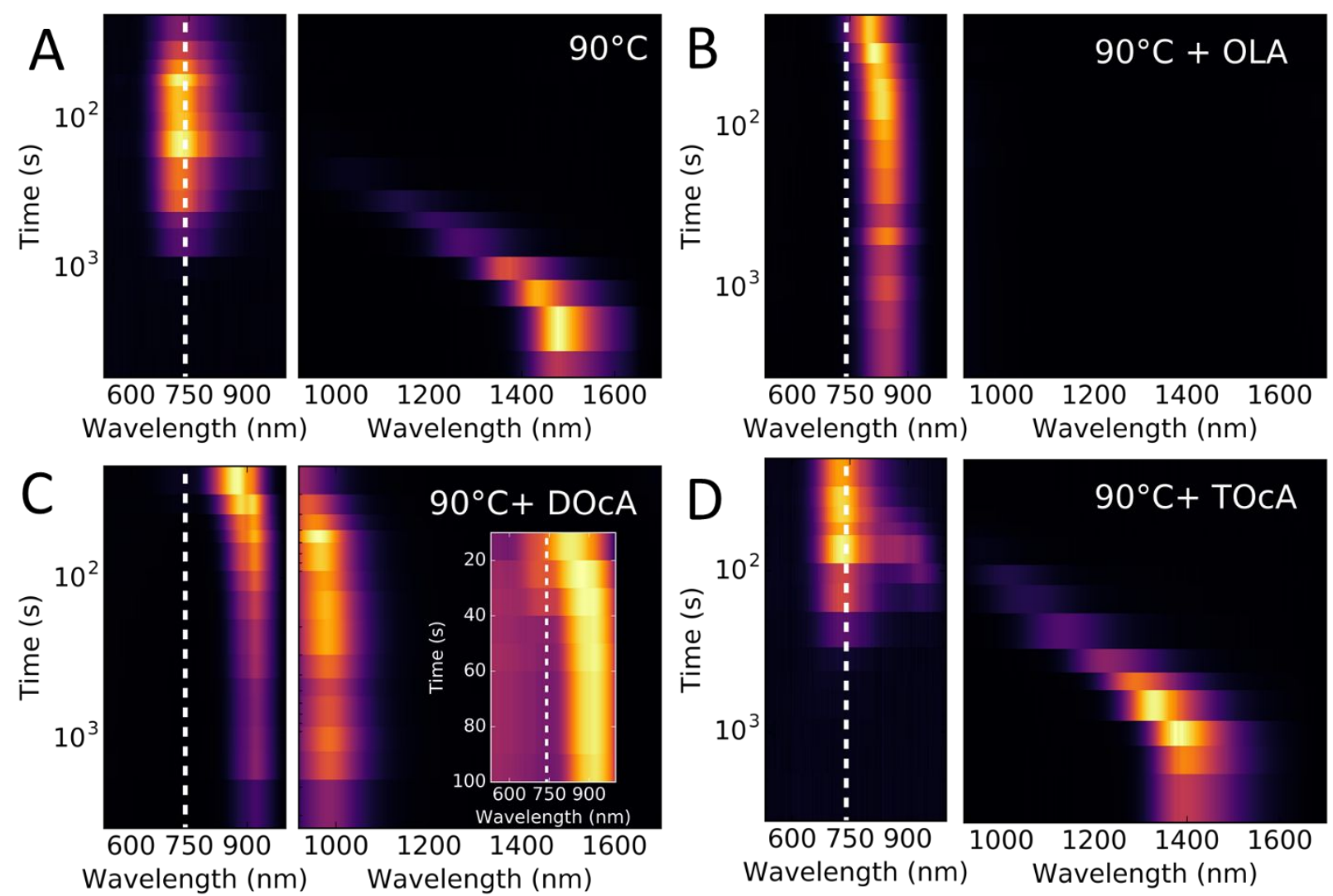

Figure S18: Influence of steric hindrance, as represented by a primary, secondary, and tertiary amine, on $\mathrm{NC}$ growth in dilute reaction coordinates (A: no amine, B: OLA, C: DOcA, D: TOcA). The inset in C) highlights emission consistent with a small concentration of PNCs at the early stages of the reaction. 


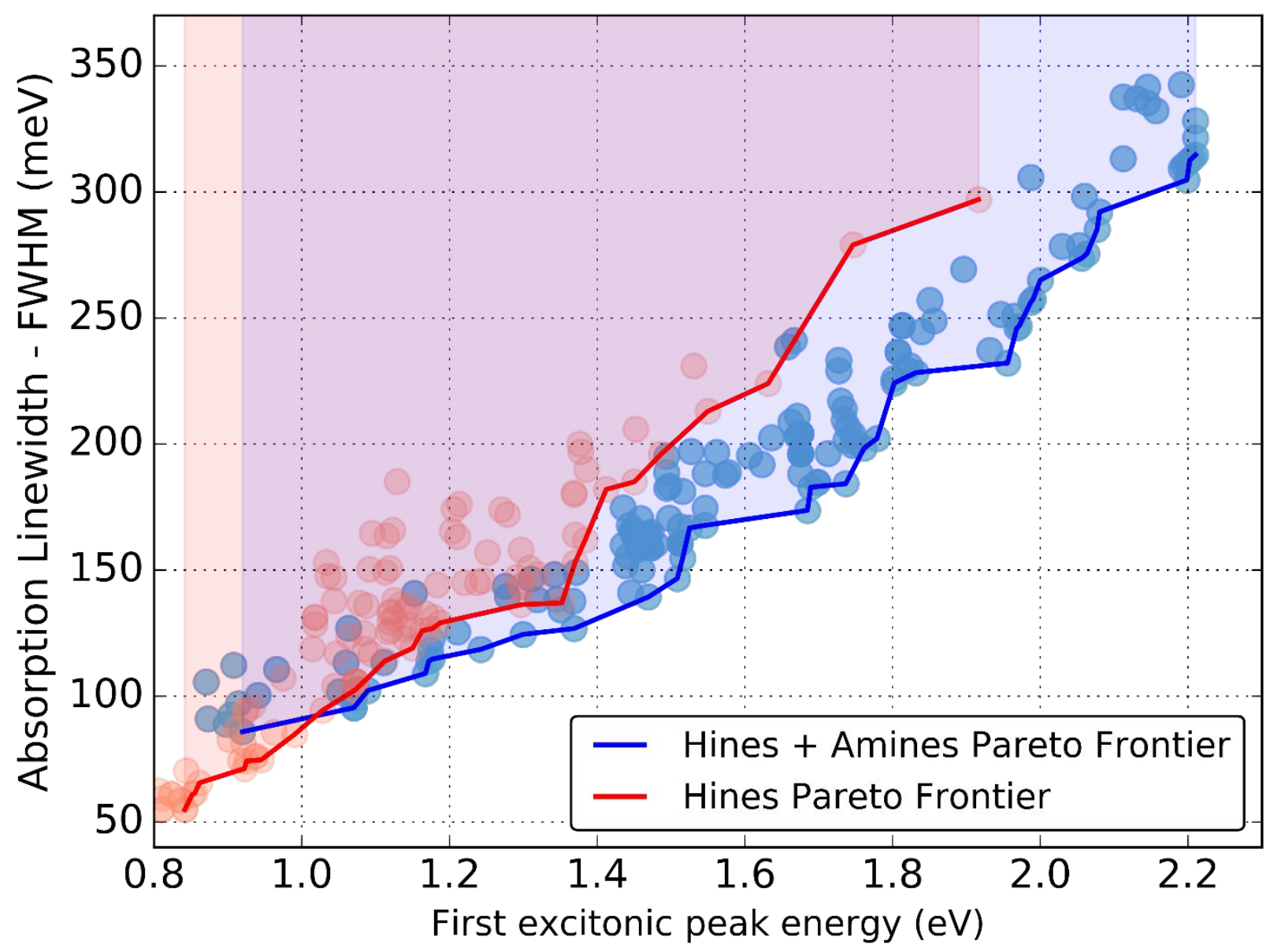

Figure S19: Absorption linewidths for all PbS NCs synthesized as part of this work, with either the classical Hines approach (red) and with added amines (blue). The Pareto Frontier highlights the best syntheses, and follows NCs that have a narrower linewidth than any smaller nanocrystal (i.e. with a larger optical gap.) The addition of amines permits narrower ensemble linewidths for NCs with excitonic first absorption peaks with energies greater than $1.1 \mathrm{eV}$, and this advantage is pronounced for bandgap energies above $1.5 \mathrm{eV}$. By contrast, the introduction of amines is detrimental for large NCs with low-energy optical gaps $(<1.0 \mathrm{eV})$, where the traditional Hines approach yields narrower linewidths. 

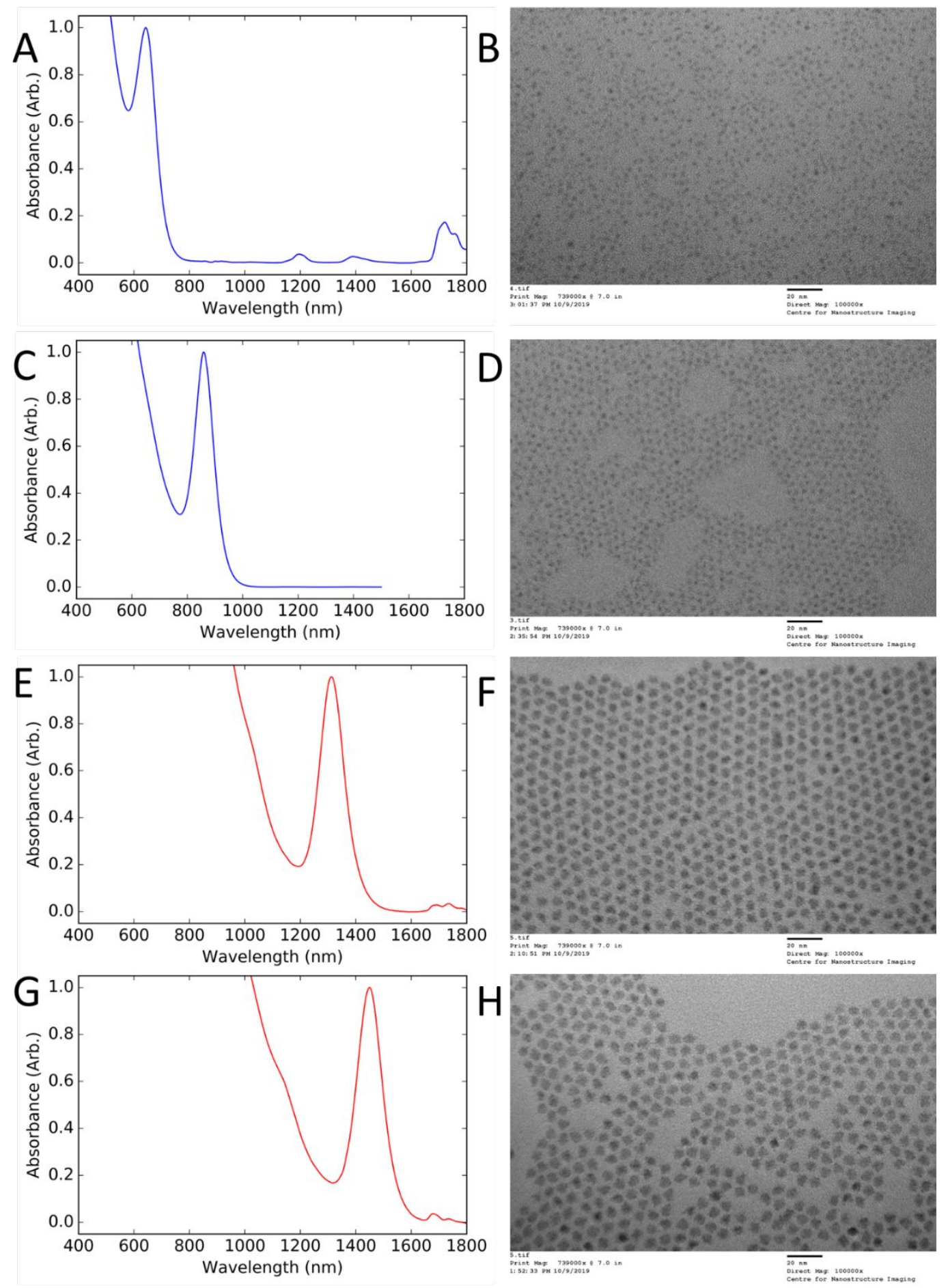

Figure S20: TEM images of representative synthesis, along with corresponding absorption spectra. See sizing histograms in figure S21 and experimental Section for Methods. 

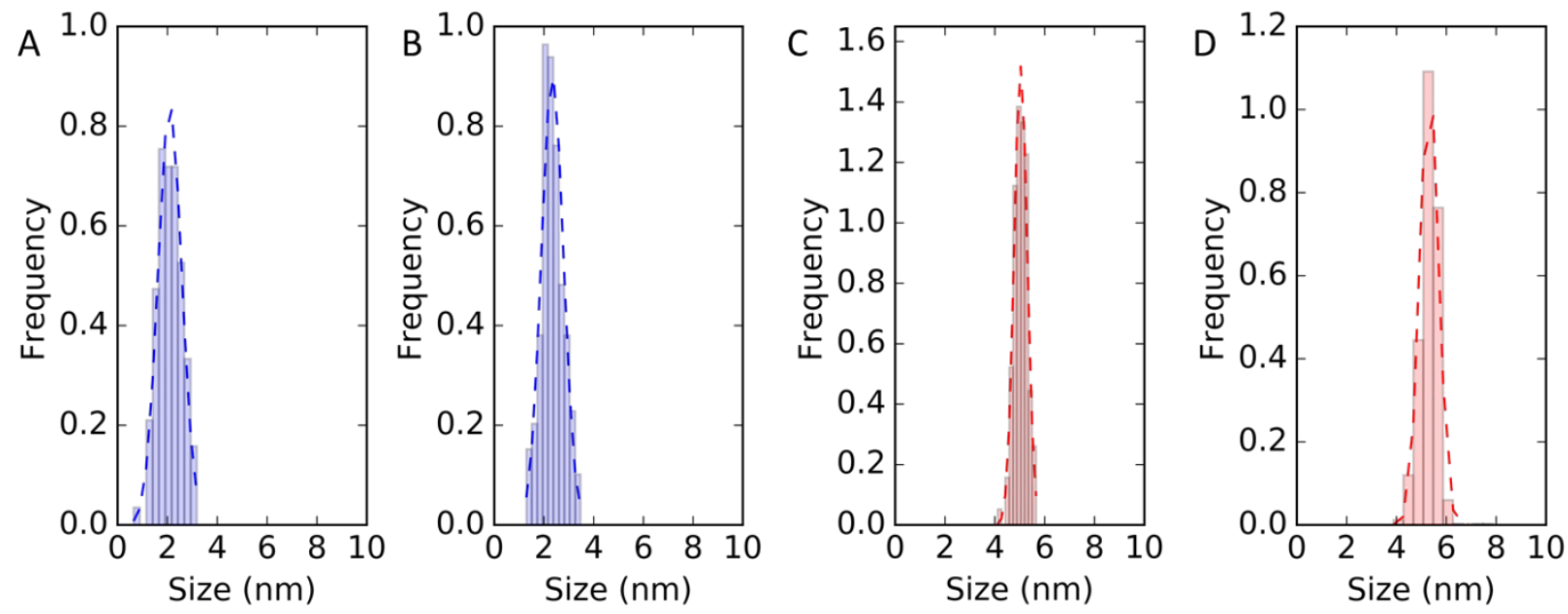

Figure S21: Representative size histograms of nanocrystal synthesis made with amines (A,B) and without (C,D). All histograms are extracted from the TEMs on Figure S20 (Panels B, D, F, \& H). The difficulties in estimating the size of such small nanocrystals (such as those illustrated in A and B) have been discussed elsewhere, stemming in part from the poor TEM contrast afforded by such small particles. ${ }^{1}$ As a result, we also used the optical linewidth to generate an additional estimate of the size dispersion (technique described below). We find, for instance, that the size dispersity extracted from TEM for the $\varnothing=2.1 \mathrm{~nm}$ nanocrystals in (A) is more than 3 times greater than expected from the optical linewidth.

Table S4: Calculated and estimated size and size dispersions of nanocrystals prepared with and without amine

\begin{tabular}{lllllllll}
\hline $\begin{array}{l}\text { Histogram } \\
\text { (Fig S21) }\end{array}$ & $\begin{array}{l}\text { UV and } \\
\text { TEM (Fig } \\
\text { S20) }\end{array}$ & $\begin{array}{l}\text { Usage of } \\
\text { amines }\end{array}$ & $\begin{array}{l}\text { Number } \\
\text { of NCs } \\
\text { sized }\end{array}$ & $\begin{array}{l}\text { Diameter } \\
(\mathbf{n m})\end{array}$ & $\begin{array}{l}\text { Standard } \\
\text { deviation } \\
\text { (nm) }\end{array}$ & $\begin{array}{l}\text { TEM Size } \\
\text { dispersion } \\
(\%)\end{array}$ & $\begin{array}{l}\text { HWHM } \\
\text { (meV) }\end{array}$ & $\begin{array}{l}\text { Size dispersion } \\
\text { estimated from } \\
\text { linewidth using } \\
\text { equation S1 (\%) }\end{array}$ \\
\hline A & A, B & Yes & 224 & 2.1 & 0.47 & 22 & 119 & 6.6 \\
\hline B & C, D & Yes & 181 & 2.3 & 0.44 & 19 & 71 & 4.3 \\
\hline C & E, F & No & 249 & 5.0 & 0.26 & 5.1 & 37 & 5.3 \\
\hline D & G, H & No & 832 & 5.3 & 0.36 & 6.8 & 31 & 4.6 \\
\hline
\end{tabular}

While the size dispersions extracted from TEM images are large for the smaller NCs, the standard deviations are smaller then lattice constant for bulk PbS $(0.5936 \mathrm{~nm})$, and are comparable or better to other distributions of small size nanocrystals. ${ }^{1,9}$ As shown summarily in Figure 5, the optical absorption of these samples exhibit ensemble linewidths that are narrower than previous reports for similar-sized nanocrystals, consistent with a smaller distribution of quantum-confined optical gaps. ${ }^{10}$ 
To estimate the size and size dispersity from the absorption peak and linewidth, we followed the approach outlined by Weidman et al. This centers on an empirical equation (S1) that relates the half width half maximum (HWHM) of the first excitonic peak to the estimated size and size dispersion. ${ }^{10}$ Equation S1 is derived assuming that the first excitonic peak is Gaussian and that the homogenous linewidth is infinitesimal (i.e. single-nanocrystal contributions to the convolution are delta functions.) This treatment is conservative for our purposes. This is because the second approximation considerably overestimates the size-dispersity for a given absorption linewidth due to the expectation that the homogenous linewidth of $\mathrm{PbS} \mathrm{NC}$ is appreciable. ${ }^{11} \mathrm{We}$ have modified the parameters of this equation to match the appropriate sizing curve for chloride-free $\mathrm{PbS} \mathrm{NCs} .{ }^{8,12}$

$$
H W H M(d, \sigma)=\frac{(0.00349 d \sigma+0.593 d+3.33) \sigma}{(0.0118 \sigma+1)(0.0252 d+0.282)(0.000281 d \sigma+0.0252 d+0.283) d}
$$

Where:

HWHM: half width half maximum of the first excitonic peak (meV)

$d$ : average diameter of the nanocrystal ensemble estimated by TEM (nm)

$\sigma:$ size dispersion $(\%$ of the diameter $)$ 


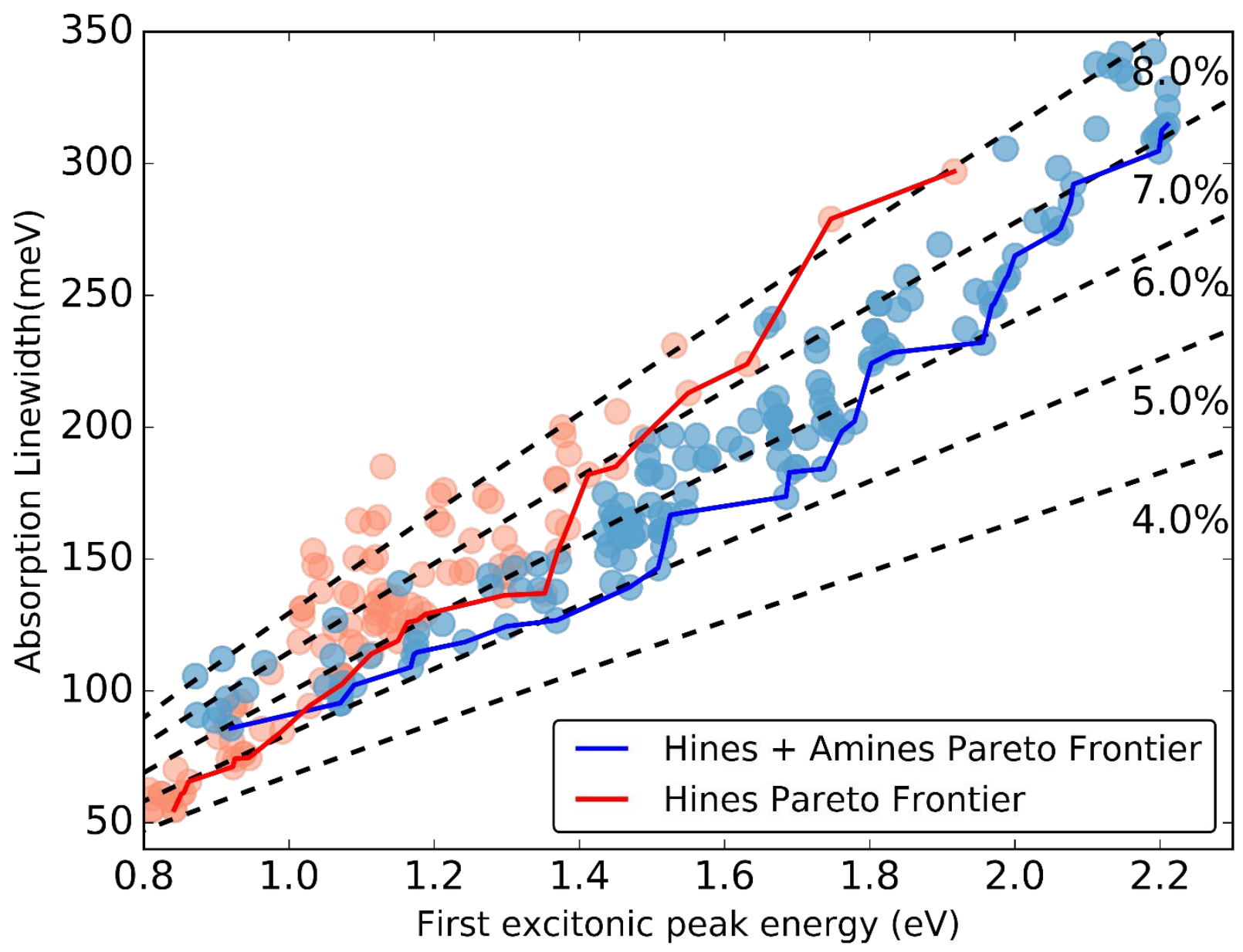

Figure S22: Observed ensemble absorption linewidths and Pareto frontiers of all $\mathrm{PbS} \mathrm{NCs}$ synthesized as part of this work (this data is identical to Figure S19.) Additional dashed lines are obtained from an empirical Equation S1, relating the absorption peak and linewidth to the nanocrystal size and size dispersion. Dashed lines represent the predicted best-achievable sizedependent ensemble linewidths for size-dispersities: $\sigma=4,5,6,7$, and $8 \%$. As described above, these curves are conservative estimates of the size-dispersity that corresponds to an observed linewidth because no homogeneous broadening is assumed (i.e. the ensemble linewidth arises entirely from size-dispersity). Nonetheless, this graph illustrates that the best nanocrystals prepared with amines are consistent with size dispersities of at most 5-7\%. 
Supplemental References:

(1) Ma, W.; Swisher, S. L.; Ewers, T.; Engel, J.; Ferry, V. E.; Atwater, H. A.; Alivisatos, A. P. Photovoltaic Performance of Ultrasmall PbSe Quantum Dots. ACS Nano 2011, 5 (10), 8140-8147. https://doi.org/10.1021/nn202786g.

(2) Choi, H.; Ko, J.-H.; Kim, Y.-H.; Jeong, S. Steric-Hindrance-Driven Shape Transition in PbS Quantum Dots: Understanding Size-Dependent Stability. Journal of the American Chemical Society 2013, 135 (14), 5278-5281. https://doi.org/10.1021/ja400948t.

(3) Hwang, G. W.; Kim, D.; Cordero, J. M.; Wilson, M. W. B.; Chuang, C.-H. M.; Grossman, J. C.; Bawendi, M. G. Identifying and Eliminating Emissive Sub-Bandgap States in Thin Films of PbS Nanocrystals. Advanced Materials 2015, 27 (30), 4481-4486. https://doi.org/10.1002/adma.201501156.

(4) Gary, D. C.; Terban, M. W.; Billinge, S. J. L.; Cossairt, B. M. Two-Step Nucleation and Growth of InP Quantum Dots via Magic-Sized Cluster Intermediates. Chemistry of Materials 2015, 27 (4), 1432-1441. https://doi.org/10.1021/acs.chemmater.5b00286.

(5) Holder, C. F.; Schaak, R. E. Tutorial on Powder X-Ray Diffraction for Characterizing Nanoscale Materials. ACS Nano 2019, 13 (7), 7359-7365. https://doi.org/10.1021/acsnano.9b05157.

(6) Hines, M. A.; Scholes, G. D. Colloidal PbS Nanocrystals with Size-Tunable Near-Infrared Emission: Observation of Post-Synthesis Self-Narrowing of the Particle Size Distribution. Advanced Materials 2003, 15 (21), 1844-1849. https://doi.org/10.1002/adma.200305395.

(7) Zhang, J.; Crisp, R. W.; Gao, J.; Kroupa, D. M.; Beard, M. C.; Luther, J. M. Synthetic Conditions for High-Accuracy Size Control of PbS Quantum Dots. The Journal of Physical Chemistry Letters 2015, 6 (10), 1830-1833.

https://doi.org/10.1021/acs.jpclett.5b00689.

(8) Moreels, I.; Lambert, K.; Smeets, D.; De Muynck, D.; Nollet, T.; Martins, J. C.; Vanhaecke, F.; Vantomme, A.; Delerue, C.; Allan, G.; et al. Size-Dependent Optical Properties of Colloidal PbS Quantum Dots. ACS Nano 2009, 3 (10), 3023-3030. https://doi.org/10.1021/nn900863a.

(9) Hou, B.; Cho, Y.; Kim, B. S.; Hong, J.; Park, J. B.; Ahn, S. J.; Sohn, J. I.; Cha, S.; Kim, J. M. Highly Monodispersed PbS Quantum Dots for Outstanding Cascaded-Junction Solar Cells. ACS Energy Letters 2016, 1 (4), 834-839.

https://doi.org/10.1021/acsenergylett.6b00294.

(10) Weidman, M. C.; Seitz, M.; Stranks, S. D.; Tisdale, W. A. Highly Tunable Colloidal Perovskite Nanoplatelets through Variable Cation, Metal, and Halide Composition. ACS Nano 2016, 10 (8), 7830-7839. https://doi.org/10.1021/acsnano.6b03496.

(11) Caram, J. R.; Bertram, S. N.; Utzat, H.; Hess, W. R.; Carr, J. A.; Bischof, T. S.; Beyler, A. P.; Wilson, M. W. B.; Bawendi, M. G. PbS Nanocrystal Emission Is Governed by Multiple Emissive States. Nano Letters 2016, 16 (10), 6070-6077. https://doi.org/10.1021/acs.nanolett.6b02147. 
(12) Green, P. B.; Li, Z.; Wilson, M. W. B. PbS Nanocrystals Made with Excess PbCl2 Have an Intrinsic Shell That Reduces Their Stokes Shift. The Journal of Physical Chemistry Letters 2019, 10 (19), 5897-5901. https://doi.org/10.1021/acs.jpclett.9b01841. 